\title{
New aspects of whistler waves driven by an electron beam studied by a 3-D electromagnetic code
}

\author{
Ken-Ichi Nishikawa ${ }^{1}$, Oscar Buneman ${ }^{2,3}$, and Torsten Neubert ${ }^{4}$
}

\begin{abstract}
We have restudied electron beam driven whistler waves with a 3-D electromagnetic particle code. The simulation results show electromagnetic whistler wave emissions and electrostatic beam modes like those observed in the Spacelab 2 electron beam experiment. It has been suggested in the past that the spatial bunching of beam electrons associated with the beam mode may directly generate whistler waves. However, the simulation results indicate several inconsistencies with this picture: (1) whistler waves continue to be generated even after the beam mode space charge modulation looses its coherence, (2) the parallel (to the background magnetic field) wavelength of the whistler wave is longer than that of the beam instability, and (3) the parallel phase velocity of the whistler wave is smaller than that of the beam mode. The complex structure of the whistler waves in the vicinity of the beam suggest that the transverse motion (gyration) of the beam and background electrons is also involved in the generation of whistler waves.
\end{abstract}

\section{Introduction}

During the Spacelab 2 flight, launched on July 29, 1985, the Plasma Diagnostics Package (PDP) performed a fly-around of the shuttle at a distance of up to $300 \mathrm{~m}$ while an electron beam was being ejected from the shuttle in a steady (dc) mode [Gurnett et al., 1986]. The plasma waves observed as the PDP approached and crossed through the beam took the appearance of a well-defined, funnel-shaped feature when displayed on frequency-time spectrograms. The waves were observed over a broad frequency range extending up to the electron cyclotron frequency $\left(f_{c} \approx 1 \mathrm{MHz}\right)$ with the center of the funnel coinciding with the passage through the beam. The mode was identified as the whistler mode, propagating with the wave normal close to the resonance cone. At even higher frequencies (3.1 MHz), intense narrowband emissions were observed inside the beam. These emissions are believed to be either at the

\footnotetext{
${ }^{1}$ Department of Physics and Astronomy, University of Iowa

2 STAR Laboratory, Stanford University

3 Deceased January 24, 1993

${ }^{4}$ Space Physics Research Laboratory, University of Michigan
}

Copyright 1994 by the American Geophysical Union.

Paper number 94GL00695

0094-8534/94/94GL-00695\$03.00 local electron plasma frequency $f_{p}$ or the upper hybrid resonance frequency, $f_{U H R}=\left(f_{c}^{2}+f_{p}^{2}\right)^{1 / 2}[$ Gurnett et $a l ., 1986]$. A review of recent beam experiments is given by Neubert and Banks [1992].

Omura and Matsumoto [1988a,b] performed the first simulation work related to the Spacelab 2 electron beam experiment, involving the initial transient response of the plasma to the injected electron beam. They presented their results of a $2-D$ slab model and concluded that whistler waves can be generated directly by a finitesized electron beam as a result of a beam instability itself independent of the electrostatic (ES) instability.

Employing 1-D ES particle simulations, Farrell et al. [1989] found that Langmuir turbulence in the beam created a spatial modulation of the current structure, and therefore enhanced the the ability of the beam to radiate. Estimates of the radiation levels based on simulations were found to be in good agreement with observed levels of radiation. Electromagnetic simulations in 2-D supported this picture [Pritchett et al., 1989]. Even with no initial net current (a quiet start; background electrons drift opposite to beam electrons providing a return current to the spacecraft), ES beam-plasma instability is generated inside the beam creating a current structure due to the bunched beam electrons which allows for the radiation of whistler waves.

Several studies have implied that the simple picture of an electron beam, space charge modulated by Langmuir turbulence, is not the means through which whistler radiation is generated, although it may be an important "seed" mechanism to get the process started. These works include Goerke et al. [1990] who, in a soundingrocket experiment, found no evidence to support the hypothesis that ES bunching at the plasma frequency was responsible for the enhancement of the whistler mode radiation. Omura and Matsumoto [1987] studied on a general competing process of the ES and whistler instability analytically and using a 1-D electromagnetic code. They found that the whistler waves are unstable enough even after the ES instability reaches its saturation, which will be discussed in this paper. Furthermore, simulation results have pointed out that the wavelength of the electrostatic beam turbulence differs from the wavelength of the whistler wave [Omura and Matsumoto, 1988a].

We attempt to establish more insight into the generation mechanism of whistler waves using a 3-D, electromagnetic, and relativistic particle code [Buneman, 1991; Buneman et al, 1992; Neubert et al., 1992]. It extends the 3-D simulations of Nishikawa et al. [1989] by 
using a larger system and by including all components of the magnetic field as well as a self-consistent poloidal magnetic field as an initial condition.

\section{Simulation Results}

The system size used for the simulations is $L_{x}=L_{y}=$ $85 \Delta, L_{z}=160 \Delta$, where $L_{x}, L_{y}$, and $L_{z}$ are the lengths of the system in 3-D and $\Delta(=1)$ is the grid size. Periodic boundary conditions are used along the ambient magnetic field ( $z$-direction), while radiating boundary conditions [Lindman, 1975] are used in the $x$ - and $y$ directions.

The electron beam is initially in a column with radius $r_{e b}=4.47 \Delta$, and the axis at the center of the domain $\left(x_{c}=y_{c}=43\right)$ oriented in the $z$-direction. The plasma density within the beam column is the same as outside, and the beam is initially charge neutral. The location of beam electrons within the column are randomly chosen and is about half of the total electron density. The total number of beam electrons is 4899 . The beam drift velocity $V_{d}=3.5 v_{e t}$, where $v_{e t}$ is the electron thermal velocity. The beam electron temperature $T_{e b}=0.09 T_{e}$, where $T_{e}$ is the temperature of the background electrons. In this study the ion drift velocity $V_{i}$ is zero. To approximately simulate ionospheric conditions, we chose the following parameters: $m_{i} / m_{e}=64, T_{e} / T_{i}=$ $1, \Omega_{e} / \omega_{p e}=0.4, c / v_{e t}=10.67, \omega_{p e} \Delta t=0.10, \beta=$ $0.111, \lambda_{D e}=0.469 \Delta, \rho_{e}=1.17 \Delta, \rho_{i}=9.38 \Delta$, and $c / \omega_{p e}=5.00 \Delta$.

As a new feature, quiet start conditions are achieved by including an initial poloidal magnetic field (IPMF) consistent with the current density $J_{z}$ carried by the beam electrons at $t=0$. (The maximum value of this poloidal magnetic is $17 \%$ of the ambient magnetic field $\left.\left(B_{z o}=0.1\right)\right)$. The IPMF is calculated from the curl of the vector potential $\mathbf{A}$, where $\mathbf{A}=\left(0,0, A_{z}\right)$ and $A_{z}$ is given by, $A_{z}(i, j)=A_{z o} \log \left(r^{2} / r_{e b}^{2}\right) ; r \geq$ $r_{e b}, A_{z}(i, j)=A_{z o}\left(\log (1.0)+r^{2} / r_{e b}^{2}-1.0\right) ; r<r_{e b}$, where $A_{z o}=-\pi r_{e b}^{2} J_{z} /(4 \pi c), r\left(=\left(i-x_{c}\right)^{2}+\left(j-y_{c}^{2}\right)\right)$ is the radius from the beam column axis, and $J_{z}$ is the current density in the column.

The total electric and magnetic field energies as func-

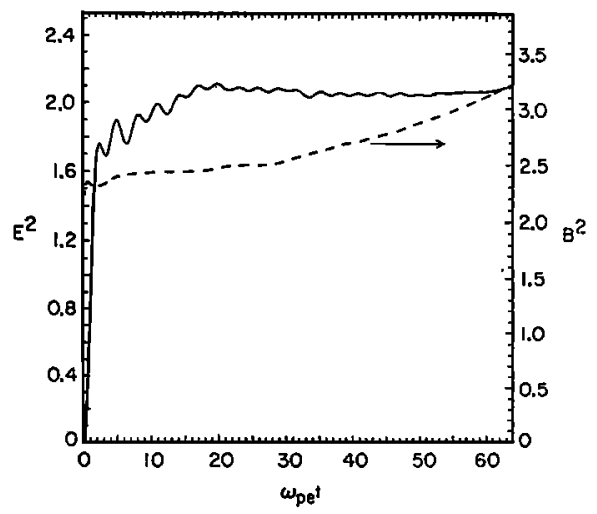

Figure 1. The time history of the electric (solid curve) and magnetic (dashed curve) field energy.
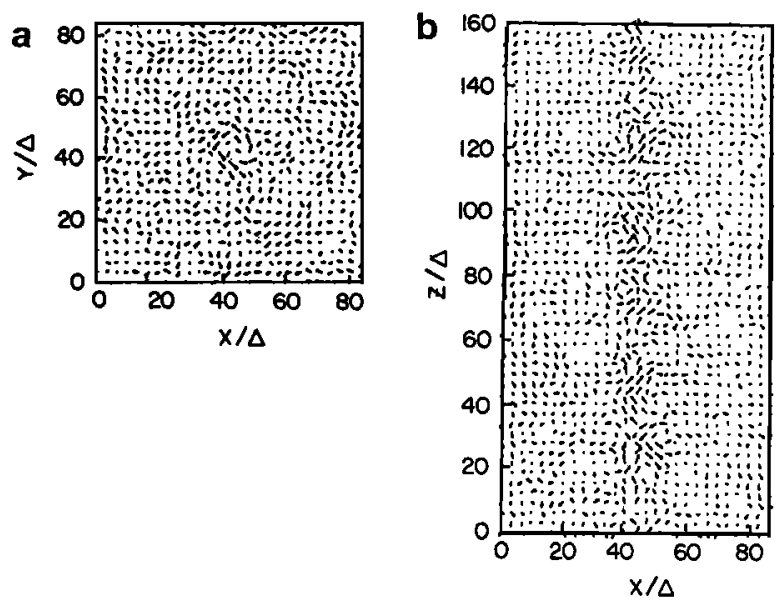

Figure 2. The fields in the 2-dimensional planes with the initial poloidal magnetic field. (a) $B_{x}-B_{y}$ in the $x-y$ plane $(z=80)$ at $\omega_{p e} t=25$, and (b) $B_{x}-B_{z}$ in the $x-z$ plane $(y=43)$ at $\omega_{p e} t=25.6$.

tions of time are shown in Figure 1. The electric field energy (solid curve) saturates around $\omega_{p e} t=20$, however, the magnetic field energy (dashed curve includes the IPMF) increases even after $\omega_{p e} t=30$, as first found by Omura and Matsumoto [1988a].

In Figure 2a, $B_{x}, B_{y}$ (with the IPMF subtracted) is shown in the center plane perpendicular to the beam axis at $\omega_{p e} t=25$. Slightly before this time the electric field energy becomes maximum. The length of the vectors have been scaled to enhance the weaker signals to better illustrate the field structure. A complicated wave pattern resembling a honeycomb structure is seen rather than simple concentric wave fronts. This structure oscillates in time (not shown) constituting an outward propagating electromagnetic wave. This is one of the new results obtained only by a 3-D simulation.

Figure $2 \mathrm{~b}$ shows the magnetic field components $B_{x}, B_{z}$ in the center $(x, z)$ plane. The ambient magnetic field $\boldsymbol{B}_{z o}$ has been subtracted. Waves are clearly created near the beam and propagating out in a complicated manner. If the parallel current is uniform, as in the initial conditions, the values of $B_{x}, B_{z}$ should be zero. These components take on finite values in the center of the beam, which leads to the idea that a poloidal structure of the current is produced in addition to the current along the $z$-direction. A similar structure is seen in our previous paper discussing the polarization of a plasma cloud [Neubert et al, 1992]. Here, space charge layers formed at the edges of the cloud generate complex current structures carried by ambient electrons. It is likely that the time-varying space charge structures generated by the bunching of beam electrons, in a similar way, generate currents in the ambient plasma that contribute to the generation of whistler waves.

The beam electrons in phase space $z-v_{z}$ is shown in Figure 3a. The electrons are bunched, accelerated, and decelerated, fundamentally due to the excited ES waves. The background electrons, initially in the column, also show bunching and acceleration in and around the beam 
a

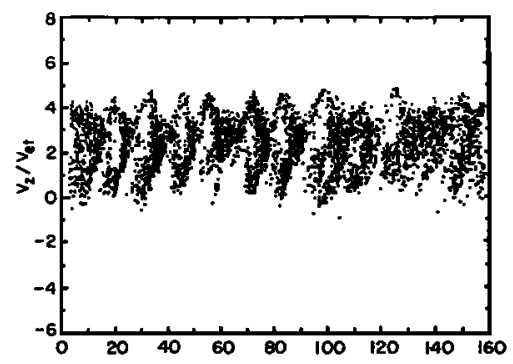

b
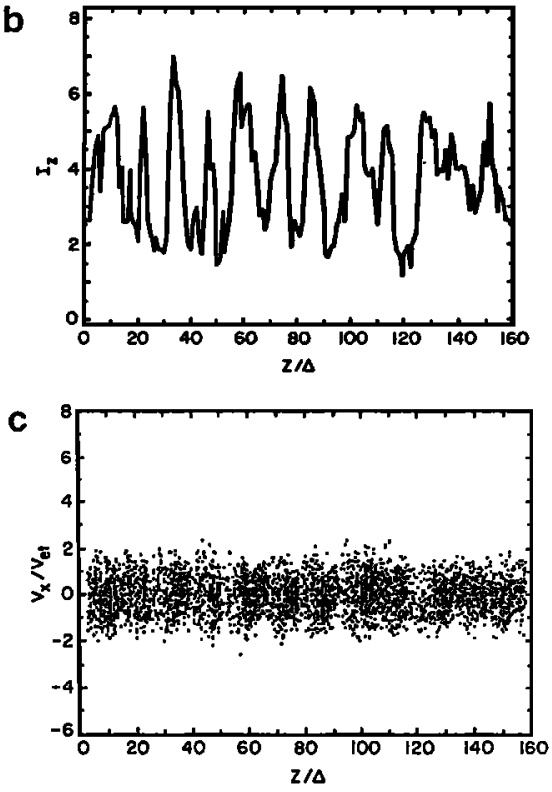

Figure 3. Snap shots of phase space distributions of the beam electrons and the total current at $\omega_{p e} t=25$. (a) The phase space in $z-v_{z}$, (b) the total current by the beam and background electrons initially in the column, and (c) the phase space in $z-v_{x}$.

column (not shown). As a result, a perturbed current structure is generated which acts like an antenna and emits whistler waves as suggested by Farrell et al. [1988, 1989], Pritchett et al. [1989], and Wong and Lin [1990]. The $J_{z}$-component of the current averaged in the column is shown in Figure 3b. Our study found that although the beam initially was directed along the ambient magnetic field with no transverse component, transverse motions develop which are essential to the whistler wave generation. The beam electrons in phase space $z-v_{x}$ are plotted (Fig. 3c). The beam electrons have been accelerated significantly in the transverse direction. We will return to this point later.

The evolution in time of the charge density and the magnetic field component $B_{x}$ along the beam axis are illustrated in Figure 4a,b. A closer inspection of Figure $4 \mathrm{a}, \mathrm{b}$ indicate that the space charge oscillations get randomized later while whistler waves continue to be generated. In fact, the different characteristics of the ES and whistler waves in phase velocities, frequencies, and wavenumbers are clearly illustrated in Figure 5a,b. As shown in Figure 5b, the whistler waves are confined below $0.5 \omega_{p e} \approx \Omega_{e}$ and less than $3 \omega_{p e} / c$. On the other hand, the ES waves are complicated and extended at higher frequencies and larger wavenumbers (Fig. 5a). They look completely different as pointed out by Omura and Matsumoto [1988a,b]. This means that to generate whistler waves a more complicated (additional) mechanism is required besides a parallel perturbed current due to the bunching of beam electrons which is consistent with the conclusion of Omura and Matsumoto [1988a.b]. It is also consistent with the idea that the self-quenching of the electrostatic instability by creation of an enhanced (turbulent) velocity spread may still allow the whistler mode to take over eventually [Bell and Buneman, 1964]. It suggests that some current structure still exists in the 9-D system (helically).

\section{Discussion}

We have attempted to establish a clearer picture of the generation mechanism of whistler waves. Results show an electrostatic mode contained within the beam column and whistler emissions propagating from the beam into the ambient plasma. These results agree qualitatively with observations obtained by PDP during the Spacelab 2 mission. The initial homogenous parallel current is perturbed through the beam mode, developing spatial inhomogeneities, which stimulate whistler radiation [Farrell et al., 1988, 1989; Pritchett et al., 1989]. On the other hand, we found that the beam mode is well separated from the whistler mode as also pointed out by 2-D simulations [Omura and Matsumoto, $1988 \mathrm{a}, \mathrm{b}$ ] and sounding-rocket experiments [Goerke et al., 1990].

Because the beam mode must be instrumental in the perturbation of the beam current, we are faced with the challenge of unifying seemingly contradictory evidence as shown in Figure 5a,b. The correlation be-
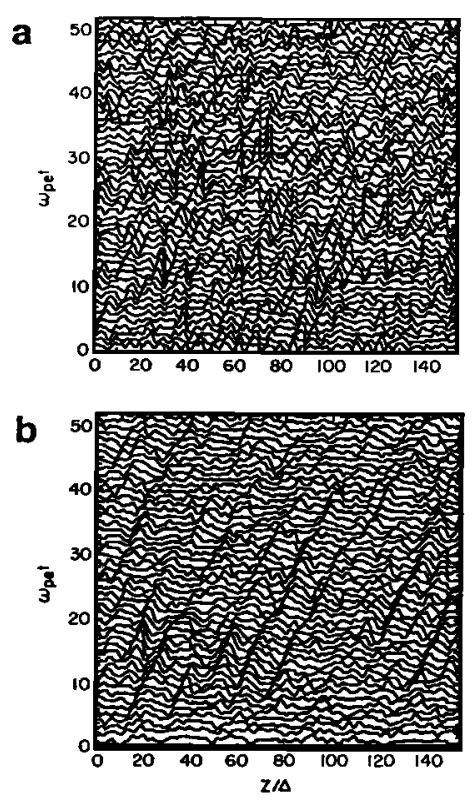

Figure 4. The time evolutions of the wave patterns along the $z$ direction at $x=43, y=43$. (a) the charge density, and (b) the magnetic field component $\left(B_{x}\right)$. 


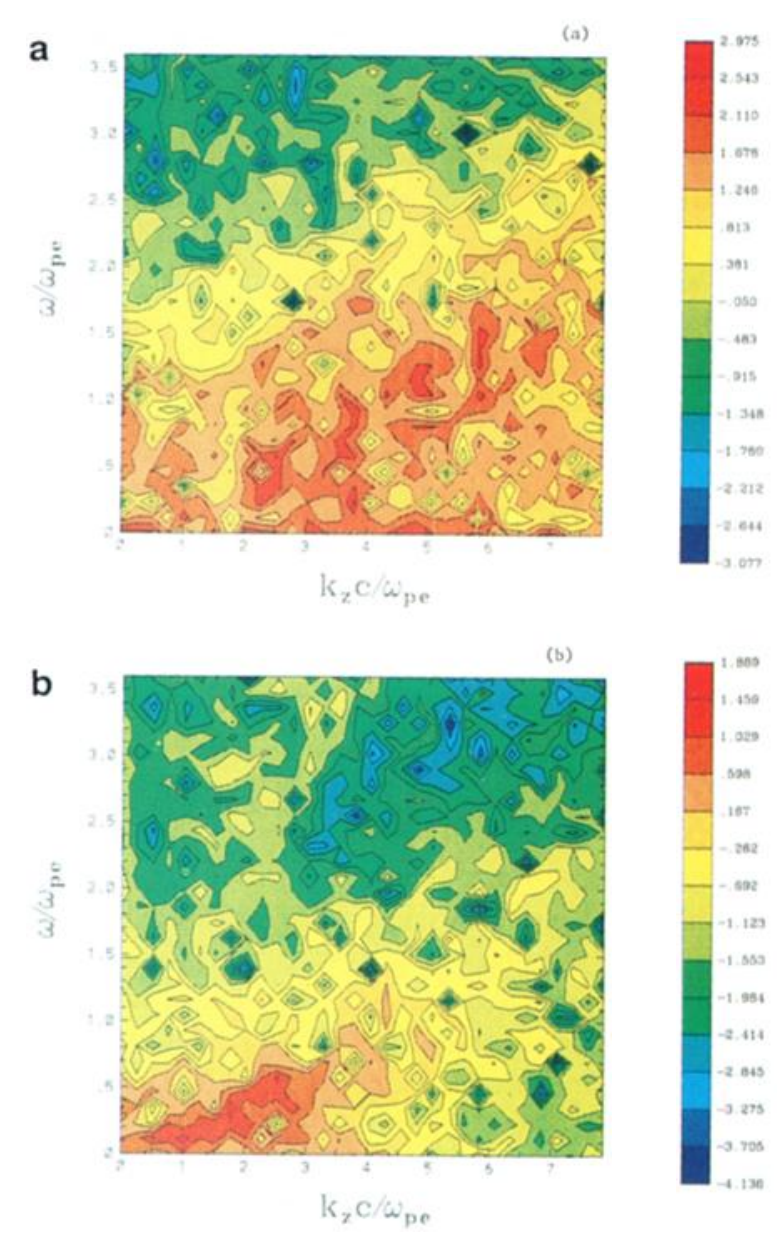

Figure 5. The wave intensities of Figure 4 as function of $\omega$ and $k_{z}$ along $22 \leq z \leq 150$ for the period $11.3<\omega_{\text {pe }} t<62.5$. (a) the charge density, and (b) the magnetic field component $\left(B_{x}\right)$.

tween the electrostatic and whistler waves at the center of the electron beam does not convince us that the parallel perturbed current due to the bunching of the beam electron generates the whistler wave by itself. We suggest a solution along the lines first discussed by Bell and Buneman [1964]. A spread in the beam electron velocity in the transverse direction leads to an instability of the stream-plasma system in the whistler mode, with the transverse electron motions serving as an energy source. As shown in Figure 2b, the magnetic component $B_{z}$ is produced by the transverse electron motion which is consistent with this finding.

Acknowledgments. Computational service was provided by the National Center for Supercomputing Applications, University of Illinois, Urbana-Champaign, which is supported by the National Science Foundation. This work was sponsored NSF under grants ATM-9106639, ATM9119814, and ATM-9121116 and by NASA under contract NAS8-38772 and grant NAGW-2350.

\section{References}

Bell, T. F. and O. Buneman, Plasma instability in the whistler mode cased by a gyrating electron stream, Phys. Rev., 133, A1300, 1964.

Buneman, O., TRISTAN: The 3-D E-M Particle Code, Proceeding of ISSS-4, Kyoto, Japan, 1991 (in press).

Buneman, O., T. Neubert, and K.-I. Nishikawa, Solar-windmagnetosphere interaction as simulated by a 3D, EM particle code, accepted to IEEE Trans Plasma Sci., 20, 810, 1992.

Farrell, W. M., D. A. Gurnett, P. M. Banks, R. I. Bush, and W. J. Raitt, An analysis of whistler-mode radiation from the Spacelab-2 electron beam, J. Geophys. Res., 93, 153, 1988.

Farrell, W. M., D. A. Gurnett, and C. K. Goertz, Coherent Cerenkov radiation from the Spacelab 2 electron beam, $J$. Geophys. Res., 94, 443, 1989.

Goerke, R. T., P. J. Kellogg, and S. J. Monson, An analysis of whistler mode radiation from a $100 \mathrm{~mA}$ electron beam, J. Geophys. Res., 95, 4277, 1990.

Gurnett, D. A. W. S. Kurth, J. J. Steinberg, P. M. Banks, R. I. Bush, and W. T. Raitt, Whistler-mode radiation from the Spacelab 2 electron beam, Geophys. Res. Lett., 13, 225, 1986.

Lindman, E. L., Free-space boundary conditions for the time dependent wave equation, J. Comput. Phys., 18, 66, 1975.

Neubert, T. and P. Bsnks, Recent results from studies of electron beam phenomena in space plasmas, Planet. Space Sci., 40, 153, 1992.

Neubert, T., R. H. Miller, O. Buneman, and K.-I. Nishikawa, The dynamics of low $-\beta$ plasma clouds as simulated by a 3-dimensional, electromagnetic particle code, J. Geophy. Res., 97, 12,057, 1992.

Nishikawa, K.-I., L. A. Frank, and C. Y. Huang, Threedimensional simulation of whistler mode excited by the Spacelab 2 electron beam, J. Geophys. Res., 94, 6855, 1989.

Omura, Y. and H. Matsumoto, Competing processes of whistler and electrostatic instabilities in the magnetosphere, J. Geophys. Res. 92, 8649, 1987.

Omura, Y. and H. Matsumoto, Computer experiments on whistler and plasma wave emissions for Spacelab-2 electron beam, Geophys. Res. Lett., 15, 319, 1988a.

Omura, Y. and H. Matsumoto, Electromagnetic and electrostatic emissions from a thin electron beam in space plasma, in Numerical Simulation of Space Plasma edited by B. Lembège and J. W. Eastwood, p. 133, NorthHolland, Amsterdam, 1988b.

Pritchett, P. L. H. Karimabadi, and N. Omidi, Generation mechanism of whistler waves produced by electron beam injection in space, Geophys. Res. Lett., 16, 883, 1989.

Wong, H. K. and C. S. Lin, Plasma instabilities of a finiteradius electron beam in a uniform plasma, Radio Sci., 25, 277, 1990.

Ken-Ichi Nishikawa, Department of Physics and

Astronomy, The University of Iowa, Iowa City, IA 52242

Torsten Neubert, Space Physics Research Laboratory, The University of Michigan, Ann Arbor, MI 48109

(received August 6, 1993; revised December 29, 1993; accepted February 18, 1994.) 